\title{
Crescimento físico de escolares de Florianópolis, Santa Catarina, Brasil: um estudo caso-controle
}

\author{
Physical growth in schoolchildren from \\ Florianópolis, Santa Catarina State, Brazil: \\ a case-control study
}

Arlete Catarina Tittoni Corso 1

Keiko Ogura Buralli 2

José Maria Pacheco de Souza 3

\footnotetext{
1 Departamento de Nutrição, Centro de Ciências Sociais, Universidade Federal de Santa Catarina. Campus Universitário da Trindade, C. P. 476 , Florianópolis, $S C$ 88040-900, Brasil. actcorso@intercorp.com.br 2 Departamento de Saúde Materno-infantil, Faculdade de Saúde Pública, Universidade de São Paulo. Av. Dr. Arnaldo 715, São Paulo, SP 01246-904, Brasil. 3 Departamento de Estatística e Epidemiologia Faculdade de Saúde Pública, Universidade de São Paulo. Av. Dr. Arnaldo 715, São Paulo, SP 01246-904, Brasil.
}

\begin{abstract}
This study was undertaken using a case-control, prevalence survey design, aimed at verifying the effects of socioeconomic, environmental, and biological/morbidity variables on the growth of schoolchildren from low-income families. The study focused on schoolchildren whose heightlage (H/A) ratio was $\leq 2 S D$ (NCHS). Control groups were paired according to sex into two groups: C1, whose H/A ratio was between -1SD (NCHS) and the median, and C2, with the H/A ratio $>$ median to $+1 S D$ (NCHS). The Mantel-Haenszel test was used to verify each variable with regard to the H/A ratio, while non-conditional multivariate analysis was used to identify which of the variable blocks had a significant effect and, in the following stage, to identify the variables with a significant effect within each block. In the first stage, the variables with a significant effect for C1 were socioeconomic. For C2, socioeconomic, environmental, and morbidity/biological variables were significant. In the final model, the remaining significant variables for C1 were socioeconomic, while those for C2 were socioeconomic and morbidity/biological. The authors conclude that socioeconomic variables are hierarchically superior to other risk factors.
\end{abstract}

Key words School Health; Body Height; Nutritional Status; Risk Factors

Resumo O estudo foi elaborado sob a forma de um desenho tipo caso-controle com base em levantamento de prevalência para verificar os efeitos das variáveis sócio-econômicas, ambientais e de morbidade/biológicas no crescimento de escolares de famílias carentes. Os casos foram escolares com indice estatura/idade (E/I) $\leq 2 \mathrm{DP}$ (NCHS). Os controles foram pareados segundo sexo em dois grupos: C1 com índice E/I entre -1 DP e a mediana, e C2 com índice E/I > mediana até + 1 DP. Utilizou-se o teste de associação Mantel-Haenszel para verificar a relação de cada variável com o índice E/I; a análise multivariada não condicional para identificar qual dos blocos de variáveis teria efeito significativo, da mesma forma que quais as variáveis teriam efeito significativo em cada bloco. Na 1a etapa, as variáveis com efeito significativo $(p<0,05)-C 1$ foram as sócio-econômicas e C2, as sócio-econômicas, as ambientais e as morbidade/biológicas. No modelo final, permaneceram com efeito significativo - C1 somente variáveis sócio-econômicas e C2, as sócio-econômicas e as morbidade/biológicas. Concluiu-se que as variáveis sócio-econômicas são hierarquicamente superiores a outros fatores de risco.

Palavras-chave Saúde Escolar; Estatura; Estado Nutricional; Fatores de Risco 


\section{Introdução}

A existência de diferenças estruturais, de variações sócio-econômicas, ambientais e culturais na sociedade se reflete nas condições desiguais de vida da população e aponta para o fato de que o processo de crescimento - em particular, a estatura alcançada pelo indivíduo na idade adulta - não deixa de ser o resultado do ambiente sociocultural integrado com outros componentes do meio, sendo que o potencial de crescimento de indivíduos de populações diferentes é similar sob condições sócio-econômicas e ambientais adequadas (Eveleth \& Tanner, 1976; Bianculli, 1985).

Para o entendimento dos fatores de risco relacionados com a desnutrição protéico-calórica entre crianças que vivem em condições sócio-econômicas e ambientais desfavoráveis, há necessidade de um modelo conceitual para explicar as relações entre os fatores de risco a serem estudados.

Atualmente existem discussões sobre a importância da utilização do modelo de análise multivariada em estudos epidemiológicos para verificação da associação entre variáveis sócioeconômicas, ambientais, reprodutivas maternas, nutricionais e demográficas (Fuchs et al., 1996; Victora et al., 1997).

Alguns autores partem do pressuposto de que, mesmo em comunidades pobres, há diferenciais sócio-econômicos que são hierarquicamente superiores a outros fatores de risco, os quais podem agir direta ou indiretamente sobre o estado nutricional infantil (Olinto et al., 1993). O modelo hierarquizado das relações entre fatores de risco para a desnutrição de Beghin et al. (1988) e Valente (1986) coloca, em primeiro lugar, os fatores sócio-econômicos, renda familiar, escolaridade dos pais, posse de utilidades domésticas e trabalho materno; em segundo lugar, os fatores reprodutivos (ordem de nascimento, intervalo interpartal e idade materna) e os fatores ambientais (moradia, aglomeração, saneamento e tipo de bairro), seguidos, em ordem de importância, do peso ao nascer, da amamentação, dos cuidados maternos e da morbidade (hospitalização, diarréia, tosse e febre).

De acordo com este modelo explicativo, depois das variáveis sócio-econômicas viriam os fatores reprodutivos e ambientais, que podem interferir nas variáveis peso ao nascer, amamentação, morbidade, estado de saúde e nutrição da criança e, consequentemente, em seu processo de crescimento. Os fatores biológicos, como idade e sexo, exercem influência sobre todas as variáveis (Olinto et al., 1993). Este mo- delo define as variáveis sócio-econômicas como hierarquicamente superiores a outros fatores de risco, principalmente quando se trata de populações aparentemente expostas a condições sócio-econômicas e ambientais semelhantes.

Atualmente, muitos estudos epidemiológicos nutricionais são realizados com crianças na idade escolar, uma vez que é nessa idade que se pode melhor avaliar a desnutrição pregressa acumulada nos últimos 7 e 8 anos, sendo que a desnutrição por déficit estatural passa a ser um reflexo do déficit de crescimento no passado de difícil reversão (Olinto et al., 1993).

O objetivo deste trabalho foi verificar a associação entre variáveis sócio-econômicas, ambientais e de morbidade/biológicas e o crescimento de escolares de uma população carente no Município de Florianópolis, Santa Catarina que estão ingressando na rede básica de ensino, para verificar se as variáveis sócioeconômicas neste município são hierarquicamente superiores a outros fatores de risco para o crescimento físico dos referidos escolares.

\section{Material e métodos}

\section{Delineamento do estudo}

Este estudo foi planejado sob a forma de um desenho tipo caso controle em um levantamento de prevalência (Mantel \& Haenszel, 1959; Schlesselman, 1982), com a finalidade de verificar fatores associados com a desnutrição por déficit estatural de escolares que estão ingressando na rede básica de ensino do Município de Florianópolis, Estado de Santa Catarina.

\section{Local do estudo}

O Município de Florianópolis, capital do Estado de Santa Catarina, conta com uma população de 271.281 habitantes, caracterizando-se por uma economia terciária e tornando-se palco de um processo migratório de famílias procedentes do interior do Estado de Santa Catarina e de outros Estados para a capital na última década, o que trouxe, como conseqüência, a instalação de 46 áreas carentes, as quais abrigam em torno de $12 \%$ do total da população do município. O Instituto de Planejamento Urbano de Florianópolis (IPUF) classifica como áreas carentes aqueles bolsões populacionais em que a grande maioria das famílias residentes possui renda média entre 0 a 3 salários mínimos. Essas áreas, além da carência econômica, apresentam problemas no setor de habitação e 
infra-estrutura sanitária e de equipamentos urbanos (IPUF, 1996).

\section{Obtenção dos dados antropométricos}

No 1o trimestre de 1994, foi realizada uma pesquisa com tomada dos dados antropométricos, para verificar a prevalência de desnutrição por déficit estatural dos escolares que ingressaram em março daquele ano na primeira série da rede básica de ensino do município. O número total de escolas de primeiro grau era de 106 , sendo $81(76,4 \%)$ escolas públicas e $25(23,6 \%)$ escolas particulares. Do total de escolas públicas, $53,1 \%$ eram localizadas na zona urbana e $46,9 \%$ na área rural do município. Todas as escolas particulares estavam localizadas na área urbana. A população de alunos que ingressavam na rede de escolas de primeiro grau totalizava 8.237 crianças, na faixa etária entre 6 a 8 anos de idade.

A pesquisa foi realizada em 95 escolas, uma vez que não se obteve permissão para efetuá-la em oito escolas particulares, além de três escolas públicas de pequeno porte terem sido fechadas. O número total de escolares foi de 4.591, pois alguns não participaram da mesma em razão de falta, doença e evasão. Do total de 3.591 escolares, 2.073 residiam em áreas carentes. Foram tomadas medidas de estatura de todos os escolares, utilizando-se fitas métricas japonesas importadas com escala milimétrica e esquadros de madeira sob os quais a criança colocava-se em posição. A tomada de medida foi feita duas vezes, e essa leitura era feita com atenção e registrada em questionário padronizado com espaço previamente destinado para isso na ficha de levantamento antropométrico.

Nas fichas da secretaria da escola tinham sido coletados anteriormente os dados sobre nome e tipo de escola, nome do escolar, data de nascimento, sexo, endereço do escolar e data da coleta de dados. Estes dados foram anotados em questionários padronizados e previamente elaborados, nos quais foi anotado posteriormente o resultado da tomada da medida de estatura de cada escolar. A coleta de todos esses dados foram realizadas por uma equipe de dez estudantes do curso de graduação em Nutrição da Universidade Federal de Santa Catarina (UFSC), previamente treinados, e a qualidade das informações foi continuamente examinada.

Foram considerados com desnutrição por déficit estatural, aqueles escolares cujos valores de estatura para idade fossem inferiores a -2 desvios-padrão em relação à população de referência do National Center for Health Statisitics - NCHS (WHO, 1995).
Após realizada avaliação nutricional, dos 4.591 escolares, encontrou-se uma prevalência de $3,1 \%$ de desnutrição por déficit estatural na população geral. Ao separar-se os escolares por áreas, verificou-se que, do total de 2.518 escolares residentes em áreas não carentes, 2,3\% apresentaram desnutrição por déficit estatural e, do total de 2.073 escolares residentes em áreas carentes, $4,1 \%$ apresentaram desnutrição por déficit estatural. A seleção dos casos e controles foi obtida a partir dos escolares residentes nas áreas carentes.

\section{Seleção de casos e controles}

O escolar residente em área carente do Município de Florianópolis, Santa Catarina que, tendo participado da pesquisa em 1994, apresentou desnutrição por déficit estatural foi considerado como caso. A presença deste déficit foi caracterizada pelo valor do índice estatura/idade $\leq$ a -2 desvios-padrão.

Foi considerado controle potencial todo escolar que, tendo participado da pesquisa de 1994, apresentou valor do índice estatura/idade $\geq$ a - 1 desvio-padrão. Os controles foram selecionados de forma a terem distribuição semelhante aos casos segundo sexo. Foram formados dois grupos de controles que originaram duas composições de bancos de dados: (a) grupo controle 1 (C1); (b) escolares cuja estatura estava entre menos um desvio-padrão e a mediana da população de referência (NCHS) e grupo controle 2 (C2); (c) escolares cuja estatura estava entre a mediana da população de referência (NCHS) até mais um desvio-padrão. Os controles, portanto, foram selecionados de forma a comporem um grupo com estatura abaixo da mediana e um grupo com estatura acima da mediana da população de referência (NCHS, 1978).

\section{Seleção dos escolares para a amostra}

Dos 4.591 escolares examinados em 1994, 2.073 foram identificados como moradores de áreas carentes do município. Destes, 84 apresentaram valores de escore $Z$ para o índice estatura/idade $\leq$ a -2 e, dessa forma, foram considerados elegíveis para participarem do presente estudo na condição de casos.

Para compor os grupos de controle, inicialmente foram selecionados os 1.334 escolares situados nas faixas de $-2 \leq$ escore $Z \leq 1$. Esses escolares foram posteriormente divididos em dois estratos segundo os valores de escore $\mathrm{Z}$. Em cada estrato, uma amostra composta de 144 escolares foi obtida por sorteio, respeitando-se a proporcionalidade observada entre os casos 
segundo sexo; assim, considerando-se a proporção de dois controles para cada caso, fixouse $a$ priori em $5 \%$ a probabilidade de cometer um erro de primeira espécie e, sendo $35 \%$ a proporção de crianças expostas entre os controles (na população em geral, como substituto), obteve-se, para uma amostra total de 360 escolares, um poder de teste de $70 \%$ para detectar uma odds ratio mínima de 1,68 em cada estrato. Dessa forma, a amostra foi composta por 72 casos, 144 controles 1 e 144 controles 2 , totalizando 360 escolares.

\section{Entrevistas e questionário}

Em 360 domicílios, foram realizadas entrevistas com as mães ou responsáveis pelos escolares pertencentes a amostra, no período entre julho de 1994 a abril de 1995. As entrevistas foram levadas a efeito por dez estudantes do curso de graduação em Nutrição da UFSC, que haviam participado da pesquisa nas escolas. Nessa equipe de entrevistadores, constavam bolsistas do PET/CAPES (Programa Especial de Treinamento/Coordenação de Aperfeiçoamento de Pessoal de Nível Superior), com monitoria e estágio/ UFSC, todos remunerados. O questionário utilizado na entrevista era padronizado e composto por questões pré-codificadas para obtenção dos seguintes dados: tempo em que a família residia no local e procedência, condições sócio-econômicas (variáveis: renda mensal familiar, escolaridade do pai e da mãe, se o escolar esteve anteriormente matriculado em programa de suplementação alimentar - PSA; se o escolar havia freqüentado instituições de atendimento ao pré-escolar: creches e berçários), características ambientais (variáveis: disponibilidade de água tratada, destino adequado do lixo e rede pública de esgoto presentes no domicílio), morbidade/biológicas (variáveis: hospitalização por diarréia antes dos dois anos de idade, peso ao nascer e tempo de amamentação ao seio).

Para todas as variáveis foram considerados três níveis de exposição: exposto, moderadamente exposto e basal. Para a variável "tempo de amamentação ao seio”, utilizou-se a técnica de tábua de vida atuarial para obtenção do tempo mediano de aleitamento (entre casos e controles), definindo-se desta forma, o ponto de corte de 06 meses entre os níveis de exposição.

As variáveis renda mensal familiar, peso ao nascer e escolaridade do pai e da mãe foram introduzidas no modelo na forma categórica indicadora (exposto, moderadamente exposto e basal). As demais variáveis foram incluídas no modelo na forma dicotômica (exposto e basal).
Considerou-se a variável hospitalização por diarréia antes dos dois anos de idade porque é um processo infeccioso que pode levar a criança a intensa perda de peso e, consequentemente, perda de estatura. Indagou-se sobre o fato de a criança ter sido internada com problemas de diarréia neste período mais de três vezes. A diarréia é uma das principais causas de doença e motivo de consultas das crianças menores de dois anos de idade dos países em desenvolvimento, sendo que muitos autores evidenciam a relação da mesma com o baixo nível sócio-econômico da família (Victora et al., 1989; Vázquez et al., 1999).

\section{Processamento dos dados}

Os dados antropométricos foram processados através do uso do software Epi-info/Epinut (Dean et al., 1994). Na criação do banco de dados e realização da análise univariada, foram utilizados os softwares Epi-info versão 6.02 (Dean et al., 1994) e Egret (Statistics and Epidemiology Research Corporation, 1988). Para a análise multivariada, foram utilizados os softwares Egret (Statistics and Epidemiology Research Corporation, 1988) e Stata (Stata Corporation, 1997). A digitação do banco de dados foi feita pela autora principal deste trabalho, sendo continuamente analisada. A análise dos dados foi efetuada por três profissionais, sendo dois deles autores deste trabalho, no Departamento de Estatística e Epidemiologia da Faculdade de Saúde Pública, da Universidade de São Paulo (USP).

\section{Análise dos dados}

Foram feitos testes de associação (MantelHaenszel) para cada variável isoladamente, a fim de verificar a possível associação com a variável condição (caso-controle). Estimativas de odds ratio, por ponto e por intervalo foram obtidas para todas as variáveis no estudo. Para análise multivariada selecionaram-se as variáveis que apresentaram significância estatística $(\mathrm{p}<0,05)$ na análise univariada em pelo menos um grupo de controles.

A análise multivariada foi realizada de acordo com o modelo hierárquico definido na Figura 1 para a composição das variáveis, procedendo-se à análise não condicional para todas as composições do estudo.

Três blocos foram considerados no modelo, cujas variáveis apresentaram significância estatística $(\mathrm{p}<0,05)$ na análise univariada mediante o teste de associação (Mantel-Haenszel): variáveis sócio-econômicas, ambientais e morbidade/biológicas. 
O processo de modelagem foi iniciado com o modelo saturado, ou seja, incluindo os três blocos de variáveis previamente selecionados (sócio-econômicas, ambientais e morbidade/biológicas). Foram eliminados do modelo os blocos cuja saída não alterasse a "capacidade explicativa do modelo".

O objetivo desta análise foi verificar quais dos três blocos de variáveis seria significante. Uma vez identificados, o mesmo procedimento foi feito para identificar quais as variáveis dos blocos permaneceriam significantes para o modelo final.

A significância estatística referente à introdução de cada variável no modelo foi avaliada mediante o teste de razão de verossimilhança, e o ajuste do modelo como um todo, mediante a estatística "deviance", utilizando-se níveis de significância 0,05 (Hosmer \& Lemeshow, 1989).

\section{Resultados}

Dentre os 360 escolares pertencentes a amostra, $58,3 \%$ eram do sexo masculino e $41,7 \%$ eram do sexo feminino. Verificou-se em relação ao tempo em que a família residia no local, que $12,5 \%$ das famílias dos escolares com desnutrição por déficit estatural residiam no local a menos de um ano, em relação a 5,6\% e 2,0\% dos grupos de controle 1 e 2 , respectivamente. Com relação ao local de procedência, observou-se que, dentre as famílias dos escolares com desnutrição por déficit estatural, $6,9 \%$ vieram do interior do Estado de Santa Catarina em relação a $6,3 \%$ e 4,9\% dos grupos controle 1 e 2, respectivamente, e $4,1 \%$ das famílias de escolares com desnutrição por déficit estatural vieram de outros Estados, em relação a $0,7 \%$ e $3,5 \%$ dos grupos controle 1 e 2 , respectivamente, evidenciando, de certa forma, uma relação entre o processo migratório recente que vem acontecendo no Município e o processo de desnutrição sofrido pela criança desde seu nascimento, expressada nos déficits estaturais, no momento em que ingressa na escola.

Encontrou-se prevalência de 3,1\% de desnutrição por déficit estatural entre os 4.591 escolares estudados e $4,1 \%$ (84) entre os 2.073 escolares cujas famílias residiam em áreas carentes do município. Dos 84 escolares elegíveis na condição de casos, 12 (14,3\%) não participaram da pesquisa, sendo dois por motivo de recusa da mã e em responder o questionário, oito porque a família havia mudado para outras cidades e dois escolares que haviam falecido no intervalo entre a tomada de dados antropométricos na escola e a pesquisa domiciliar. O núme-
Figura 1

Modelo hierarquizado dos fatores de risco para a desnutrição por déficit estatural. Adaptado de Olinto et al. (1993).
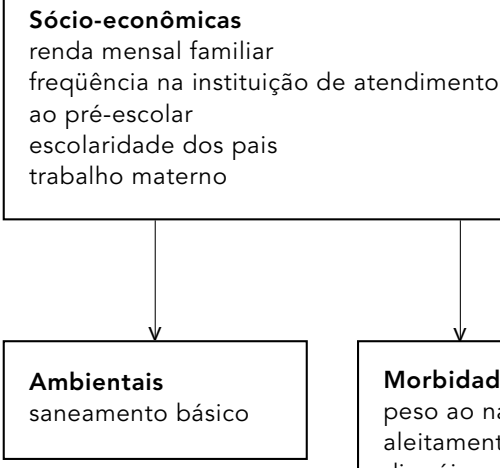

Morbidade/Biológicos

peso ao nascer

aleitamento materno

diarréia

idade

sexo

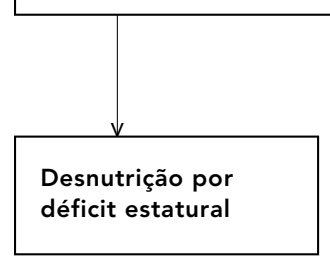

ro relativamente pequeno de casos (72 escolares) não comprometeu o estudo, pois todos os testes estatísticos planejados foram possíveis de realização. O número de 72 escolares representou uma prevalência de $3,5 \%$ com desnutrição por déficit estatural. A utilização de -2 desvios-padrão como limite implica que $2,3 \%$ da população de referência será classificada como "desnutrida" até mesmo se forem indivíduos verdadeiramente "saudáveis", sem apresentar dificuldades de crescimento. Portanto, 2,3\% podem ser considerados como "baseline" ou prevalência esperada. As prevalências em populações carentes são geralmente mais elevadas que 2,3\% (Onis \& Blössner, 1997). Como o processo de migração campo-cidade no Município de Florianópolis teve início na última década, a prevalência de desnutrição pregressa expressada na estatura dos escolares de primeira série do primeiro grau é pequena, porém acima da prevalência esperada ou "baseline".

No Brasil foram realizados alguns censos de estatura de escolares ingressantes na rede básica de ensino, utilizando-se o índice estatura/ idade. Em Osasco, São Paulo, um censo foi realizado em 1989 com cerca de 15.000 escolares, 
sendo encontrada uma prevalência de 4,8\% de desnutrição por déficit estatural (Lei, 1989). No Nordeste, em 1991 foram realizados três censos com escolares que ingressavam na primeira série do primeiro grau, para verificar a prevalência de desnutrição por déficit estatural: no Estado da Paraíba, foi encontrada uma prevalência de 17,86\% entre 7.570 escolares; no Estado do Ceará, foi encontrada uma prevalência de 17,2\% entre 63.910 escolares; no Estado do Piauí, foi encontrada uma prevalência de $28 \%$ entre 103.291 escolares (FAE/SEC-CE, 1993; FAE/SECPB, 1993; FAE/SEC-PI, 1993). Tsuyuoka et al. (1999) estudaram 360 escolares de primeiro grau de escolas públicas na capital do Estado de Sergipe, encontrando prevalência de $5,4 \%$ de desnutrição por déficit estatural. Os dados da Pesquisa Nacional sobre Saúde e Nutrição de 1989 (INAN, 1990), estudados por Engstrom \& Anjos (1999), mostram prevalência de $14,4 \%$ de desnutrição por déficit estatural no Brasil, cujas causas foram associadas com escolaridade materna, renda mensal per capita e condições de moradia.

No presente trabalho, as variáveis que apresentaram significância estatística $(\mathrm{p}<0,05)$ para o grupo controle 1 na análise univariada foram as variáveis sócio-econômicas: renda mensal familiar e freqüência em instituições de atendimento ao pré-escolar, bem como a variável de morbidade/biológica: peso ao nascer. Para o grupo controle 2, foram as variáveis sócioeconômicas: renda mensal familiar, escolaridade do pai, freqüência em instituições de atendimento ao pré-escolar, além das variáveis am- bientais: destino adequado do lixo, variáveis de morbidade/biológicas, hospitalização por diarréia, tempo de amamentação ao seio e peso ao nascer. Estas variáveis entraram na primeira etapa da análise multivariada.

No resultado da primeira etapa do processo de modelagem estatística dos dados para ambos os grupos de controles destaca-se a maior importância para a desnutrição por déficit estatural, das variáveis sócio-econômicas e de morbidade/biológicas, que, segundo critérios estabelecidos a priori, foram as variáveis selecionadas para a análise final dos dados. Incluiu-se, no conjunto de variáveis ambientais, a variável disponibilidade de água tratada por ser de importância nos estudos epidemiológicos de desnutrição infantil.

Os resultados da análise multivariada hierarquizada relacionados com a etapa final do processo de modelagem estão apresentados na Tabela 1.

Nenhuma das variáveis ambientais continuou significativa no decorrer do processo de modelagem da análise multivariada. Analisando-se o efeito conjunto de todas as variáveis, sobre o crescimento dos escolares, permaneceram significantes no modelo final (Tabela 1) para os escolares do grupo controle 1 , as variáveis sócio-econômicas renda mensal familiar (menor que dois salários mínimos (odds ratio $=6,06$ e intervalo com 95\% de confiança $=1,31$ - 12,50) e freqüência em instituições de atendimento ao pré-escolar (odds ratio $=2,80$ e intervalo com $95 \%$ de confiança $=1,05-7,47)$.

Tabela 1

Análise multivariada não condicional com as variáveis sócio-econômicas e de morbidade/biológicas.

Modelo final com todas as variáveis.

\begin{tabular}{|c|c|c|c|c|}
\hline \multirow[t]{2}{*}{ Variáveis } & \multicolumn{2}{|r|}{$\mathrm{C} 1$} & \multicolumn{2}{|r|}{$\mathrm{C} 2$} \\
\hline & odds ratio & $\begin{array}{l}\text { intervalo com } 95 \% \\
\text { de confiança }\end{array}$ & odds ratio & $\begin{array}{l}\text { intervalo com } 95 \% \\
\text { de confiança }\end{array}$ \\
\hline Sexo & 1,31 & $0,65-2,61$ & 1,77 & $0,76-4,15$ \\
\hline Renda mensal familiar (2 a 4 sm) & 2,45 & $0,95-6,30$ & 5,05 & $1,83-13,94$ \\
\hline Renda mensal familiar (<2 sm) & 4,06 & $1,31-12,59$ & 4,41 & $1,38-14,05$ \\
\hline Escolaridade do pai (1 a 8 anos) & 0,49 & $0,18-1,39$ & 0,93 & $0,32-2,64$ \\
\hline Escolaridade do pai (< 1 ano) & 0,71 & $0,17-2,87$ & 11,28 & $1,19-107,0$ \\
\hline $\begin{array}{l}\text { Freqüência a instituição de } \\
\text { atendimento ao pré-escolar }\end{array}$ & 2,80 & $1,05-7,47$ & 7,93 & $2,21-28,37$ \\
\hline Hospitalização por diarréia & 1,14 & $0,51-2,56$ & 1,61 & $0,59-4,38$ \\
\hline Peso ao nascer (2.501 a $3.000 \mathrm{~g})$ & 1,79 & $0,86-3,74$ & 4,69 & $1,86-11,85$ \\
\hline Peso ao nascer $(<2.500 \mathrm{~g})$ & 2,32 & $0,86-6,24$ & 6,35 & $1,95-20,60$ \\
\hline Tempo de amamentação & 0,85 & $0,42-1,74$ & 2,19 & $0,86-5,04$ \\
\hline
\end{tabular}

Teste de razão de verossimilhança: $C 1=215.66-\mathrm{gl}=10-\mathrm{p}=0,03 ; \mathrm{C} 2=161.54-\mathrm{gl}=10-\mathrm{p}=0,00$. 
Para os escolares do grupo controle 2, permaneceram significantes, as variáveis sócioeconômicas: renda mensal familiar (entre 2 a 4 salários mínimos (odds ratio $=5,05$ e intervalo com $95 \%$ de confiança $=1,83-13,94)$ e menos que dois salários mínimos (odds ratio $=4,41 \mathrm{e}$ intervalo com 95\% de confiança $=1,38-14,05$ ), indicando que a renda menor que quatro salários mínimos é fator de risco; escolaridade do pai (menos de um ano de estudo - odds ratio = 11,28 e intervalo com $95 \%$ de confiança $=1,19$ 107,0), freqüência em instituições de atendimento ao pré-escolar (odds ratio $=7,93$ e intervalo com $95 \%$ de confiança $=2,21-28,37$ ) e a variável de morbidade/biológica, peso ao nascer (entre $2.501 \mathrm{~g}$ a $3.000 \mathrm{~g}-$ odds ratio $=4,69 \mathrm{e}$ intervalo com $95 \%$ de confiança $=1,86-11,85$ e menos de $2.500 \mathrm{~g}$ - odds ratio $=6,35$ e intervalo com $95 \%$ de confiança $=1,95-20,60$ ), indicando que o peso menor que $3.000 \mathrm{~g}$ é fator de risco para a desnutrição por déficit estatural dos escolares residentes no Município de Florianópolis, Santa Catarina.

\section{Discussão}

Apesar da relação entre estatura e condições ambientais estar bem documentada (Kac, 1999), no presente trabalho, não foram encontradas diferenças significativas na distribuição das variáveis ambientais na etapa final da análise multivariada, pois a maioria das famílias vive em condições semelhantes, sendo que o crescimento físico dos escolares ingressantes na rede básica de ensino do Município de Florianópolis, Santa Catarina está associado aos fatores sócio-econômicos e de morbidade/biológicos.

$\mathrm{Na}$ análise de regressão logística múltipla hierarquizada, em ordem de importância, as variáveis sócio-econômicas renda mensal familiar de menos de dois salários mínimos (OR = $4,06)$ e freqüência em instituições de atendimento ao pré-escolar ( $\mathrm{OR}=2,80$ ) mostraram maiores chances para o grupo controle 1 , ou seja, aqueles escolares, cujo crescimento físico está abaixo da média em relação ao padrão de referência.

No grupo controle 2 - escolares cujo crescimento físico está acima da média em relação ao padrão de referência -, as variáveis sócioeconômicas escolaridade do pai com menos de um ano de estudo (OR $=11,28)$, freqüência em instituições de atendimento ao pré-escolar (OR = 7,93), a variável de morbidade/biológica, peso ao nascer menos de $2.500 \mathrm{~g}(\mathrm{OR}=6,35)$, nascer entre 2.501 a $3.000 \mathrm{~g}(\mathrm{OR}=4,69)$ e a variável sócio-econômica renda mensal familiar entre 2 e 4 salários mínimos $(\mathrm{OR}=5,05)$ e $<2$ salários mínimos $(\mathrm{OR}=4,41)$, foram as que permaneceram significantes relacionadas com o crescimento físico dos escolares.

No Brasil foram realizados alguns trabalhos sobre a utilização de análises multivariadas para estimar fatores de riscos de déficits estaturais, onde variáveis sócio-econômicas - como a escolaridade dos pais ao lado da renda - foram consideradas os fatores mais importantes, podendo o nível de escolaridade dos pais atuar na determinação do nível de saúde, em razão de proporcionar melhor entendimento dos mecanismos etiológicos das doenças infantis e maior eficiência nos cuidados com as crianças, além de fornecer oportunidades, melhores empregos e salários mais altos. A escolaridade paterna pode refletir a classe social, sendo um dos determinantes da renda que influenciam diretamente o consumo familiar (Monteiro et al., 1988; Hutly et al., 1991; Souza, 1992; Olinto et al., 1993).

As conclusões da pesquisa de Lei (1994), em Osasco, São Paulo, mostraram que os níveis de escolaridade do pai $(\mathrm{OR}=17,0)$ e da mãe $(\mathrm{OR}=13,8)$ destacaram-se entre os demais fatores sócio-econômicos de risco para a desnutrição por déficit estatural, evidenciando também que o efeito da escolaridade é mais intenso do que o da renda. Malta et al. (1998) realizaram estudo com 699 escolares que ingressavam no primeiro grau de escolas públicas municipais de Belo Horizonte, Minas Gerais, verificando associação entre repetência escolar, desnutrição por déficit estatural, escolaridade da mãe e renda per capita. No Estado do Ceará, Souza (1992) encontrou associação entre desnutrição por déficit estatural e escolaridade da mãe, além do peso ao nascer e da existência de instalação sanitária na residência. Guimarães et al. (1999) realizaram um estudo caso-controle em que utilizaram análise multivariada hierarquizada com 330 pré-escolares matriculados em escolas públicas do Município de Cosmópolis, São Paulo, encontrando associação entre desnutrição por déficit estatural com variáveis sócio-econômicas, dentre elas o baixo nível de escolaridade da mãe. No presente trabalho, verificou-se associação com a escolaridade paterna entre casos e grupo controle 2, assim como a renda entre casos e os dois grupos de controles.

Monteiro et al. (1992) destacam que, dentre as variáveis sócio-econômicas, a renda não é um fator absoluto, devendo ser considerados outros elementos, entre os quais o acesso da população a serviços essenciais como saúde, educação e saneamento, que, embora influen- 
ciado pelo nível de renda da família, dependem tanto dos investimentos governamentais na área social quanto da eficiência das instituições públicas e da política de prestação de serviços.

Considera-se, dentre estes investimentos, a viabilização de instituições de atendimento à criança na idade pré-escolar, principalmente nas áreas onde predominam populações de baixa renda. No presente trabalho, esta variável exerceu fator de proteção para o crescimento dos escolares $(\mathrm{OR}=7,93$ para o grupo $\mathrm{C} 2$ e $\mathrm{OR}=$ 2,8 para o grupo $\mathrm{C} 1$ ). Em estudo recente feito por Silva (1998) com 257 escolares matriculados em Centros Integrados de Educação Pública (CIEP) em Americana, São Paulo, foi encontrada uma prevalência de $15,5 \%$ de desnutrição por déficit estatural, associada com a renda mensal familiar e a freqüência em creches.

No Município de Florianópolis, encontramse creches e berçários em todas as áreas estudadas, nas quais são aceitas crianças na faixa etária de quatro meses a seis anos de idade, cujas mães trabalham fora. Essas crianças permanecem o dia todo, recebendo quatro refeições diárias. Esse conjunto de fatores exerce notável influência quando se trata de crianças residentes em áreas carentes, onde os recursos familiares a elas destinados são precários, aliado à ausência da mãe, que trabalha fora de casa.

A qualidade do serviço público destinado à população infantil, onde predomina uma população com condições sócio-econômicas e ambientais deficitárias, minimiza as conseqüências da desnutrição, na ausência de mudanças mais profundas, capazes de permitir aumento na renda mensal familiar compatível com adequada qualidade de vida.

\section{Referências}

BEGHIN, I.; CAP, M. \& DUJARDIN, B., 1988. Guia para Evaluar el Estado de Nutrición. Publicación Científica 515. Washington, D.C.: Organización Panamericana de la Salud/Organización Mundial de la Salud.

BIANCULLI, C. N., 1985. Crecimiento y desarrollo físico del adolescente. In: La Salud del Adolescente y el Joven en las Américas (Organização Panamericana de la Salud, ed.), pp. 47-53, Publicación Científica 489, Washington, D.C.: OPS.

CANTERO, M. A.; CORDIOLI, A. G. \& GALÃO, F. C., 1997. Estado nutricional das crianças de 0 a 6 anos na Unidade Básica de Saúde do Jardim União da Vitória-Londrina, PR. Semina, 189:89-96.

COSTA, C. E. \& GOTLIEB, S. L. D., 1998. Estudo epidemiológico do peso ao nascer a partir da Declaração de Nascido Vivo. Revista de Saúde Pública, 32:328-334.
Com relação à associação entre o baixo peso ao nascer e estatura, de acordo com a OMS, esta variável é o mais importante indicador das chances de um recém-nascido sobreviver e ter crescimento normal, tendo em vista sua grande influência sobre os coeficientes de mortalidade infantil e mortalidade proporcional em menores de cinco anos de idade (WHO, 1980).

No grupo controle 2 deste estudo verificouse que o peso ao nascer menor que $2.500 \mathrm{~g}(\mathrm{OR}=$ $6,35)$ e entre $2.501 \mathrm{~g}$ a $3.000 \mathrm{~g}(\mathrm{OR}=4,69)$ constitui fator de risco para a desnutrição por déficit estatural. Outros autores encontraram associação estatisticamente significante entre o baixo peso ao nascer e as condições sócio-econômicas (Fisberg et al., 1992; Cantero et al., 1997; Costa \& Gotlieb, 1998).

Neste estudo, as condições sócio-econômicas foram os fatores de risco que melhor apresentaram capacidade para predizer a ocorrência de desnutrição por déficit estatural na comparação entre os grupos de casos e controle 1 . Entre os grupos de casos e controle 2, de acordo com os resultados, além das variáveis sócioeconômicas, a variável de morbidade/biológica peso ao nascer também mostrou ser fator de risco. Essa diferença, de certa forma, explica o fato de que os escolares do grupo controle 2 , pelo fato de viverem em condições um pouco melhores, cresceram mais, atingindo estatura acima da média quando comparados com a população de referência. As diferenças nas condições de vida, por menos expressivas que sejam nas áreas carentes no Município de Florianópolis, Santa Catarina, refletem-se na estatura que as crianças atingem quando chegam à idade escolar.

DEAN, J., 1994. Epi-Info. Computer Programs for Epidemiology, Version 6.02. Atlanta: Division of Surveillance and Epidemiology Studies, Epidemiology Programs Office, Centers for Disease Control and Prevention.

ENGSTROM, E. M. \& ANJOS, L. A., 1999. Déficit estatural nas crianças brasileiras: Relação com condições sócio-ambientais e estado nutricional mateno. Cadernos de Saúde Pública, 15:559-567.

EVELETH, B. \& TANNER, J. M., 1976. Worldwide Variation in Human Growth. New York: Cambridge University Press.

FISBERG, M.; MAKSSOUDIAN, A.; CASTELLOTTI, D. S. \& JARMY, Z. I. K. A., 1992. Influência do peso de nascimento na modificação do estado nutricional. Revista Paulista de Pediatria, 10:3-6.

FUCHS, S. C.; VICTORA, C. G. \& FACHEL, J., 1996. Modelo hierarquizado: Uma proposta de mode- 
lagem aplicada à investigação de fatores de risco para diarréia grave. Revista de Saúde Pública, 30: 168-178.

FAE/SEC-CE (Fundação de Amparo ao Estudante/Secretaria de Educação e Cultura - Ceará), 1993. Primeiro censo estadual de altura/idade dos escolares de primeira série do ensino fundamental. Fortaleza: SEC-CE.

FAE/SEC-PB (Fundação de Amparo ao Estudante/Secretaria de Educação e Cultura - Paraíba), 1993. Primeiro censo estadual de altura/idade dos escolares de primeira série do ensino fundamental. João Pessoa: SEC-PB.

FAE/SEC-PI (Fundação de Amparo ao Estudante/Secretaria de Educação e Cultura - Piauí), 1993. Primeiro censo estadual de altura/idade dos escolares de primeira série do ensino fundamental. Teresina: SEC-PI.

GUIMARÃES, L. V.; LATORRE, M. R. D. G. O. \& BARROS, M. B. A., 1999. Fatores de risco para ocorrência de déficit estatural em pré-escolares. Cadernos de Saúde Pública, 15:605-615.

HOSMER, D. W. \& LEMESHOW, S., 1989. Applied Logistic Regression. New York: John Whitey \& Sons.

HUTLY, S.; VICTORA, C. G.; BARROS, F. \& VAUGHAN, J. P., 1991. The timing of nutritional status: Determination and implications for intervention and growth monitoring. European Journal of Clinical Nutrition, 45:85-95.

INAN (Instituto Nacional de Alimentação e Nutrição), 1990. Pesquisa Nacional sobre Saúde e Nutrição PNSN, 1989. Brasília: INAN.

IPUF (Instituto de Planejamento Urbano de Florianópolis), 1996. Perfil de Áreas Carentes. Florianópolis: Coordenadoria de Planejamento Habitacional, IPUF.

KAC, G., 1999. Tendência secular em estatura: Uma revisão da literatura. Cadernos de Saúde Pública, 15:451-461.

LEI, D. L. M., 1989. Coleta e Análise da Altura de Escolares em um Sistema de Vigilância Nutricional: Desenvolvimento de Metodologia, Implantação e Avaliação. Relatório Técnico. São Paulo: Faculdade de Saúde Pública, Universidade de São Paulo.

LEI, D. L. M., 1994. Retardo do Crescimento na Idade Pré-escolar: Fatores Socioeconômicos, Associação com o Estado Nutricional na Idade Escolar e Prognóstico do Aproveitamento Discente no Município de Osasco (Área Metropolitana de São Paulo). Tese de Doutorado, São Paulo: Faculdade de Saúde Pública, Universidade de São Paulo.

MALTA, D. C.; GOULART, M. E. A \& COSTA, M. F. F. L., 1998. Estado nutricional e variáveis sócio-econômicas na repetência escolar: Um estudo prospectivo em crianças da primeira série em Belo Horizonte, Brasil. Cadernos de Saúde Publica, 14:157-164.

MANTEL, N. \& HAENSZEL, W., 1959. Statistical aspects of analysis of data from retrospective studies of disease. Journal of the National Cancer Institute, 22:719-748.

MONTEIRO, C. A.; BENÍCIO, M. H. D. \& CHIEFFI, P. P., 1988. As doenças. In: Saúde e Nutrição das Crianças de São Paulo (C. A. Monteiro, org.), pp. 117141, São Paulo: Editora Hucitec/Edusp.

MONTEIRO, C. A.; BENÍCIO, M. H. D. \& GOUVEIA, N. C., 1992. Saúde e nutrição das crianças brasileiras no final da década de 80. In: Perfil Estatístico de Crianças e Mães no Brasil (M. F. G. Monteiro \& R. Cervini, org.), pp. 19-40, Rio de Janeiro: Instituto Nacional de Alimentação e Nutrição/Fundo das Nações Unidas para a Infância.

NCHS (National Center for Health Statistics), 1978. Growth Curves for Children, Birth 18 Years. United States. NCHS Series 11, no 165, Hyattsville: NCHS.

OLINTO, M. T. A.; VICTORA, C. G.; BARROS, F. C. \& TOMASI, E., 1993. Determinantes da desnutrição infantil em uma população de baixa renda: Um modelo de análise hierarquizado. Cadernos de Saúde Pública, 9(Sup. 1):14-27.

ONIS, M. \& BLÖSSNER, M., 1997. WHO Global Database on Child Growth and Malnutrition. Geneva: World Health Organization.

SCHLESSELMAN, J. J., 1982. Case Control Studies Design, Conduct and Analysis. New York: Oxford University Press.

SILVA, M. V., 1998. Estado nutricional de alunos matriculados em escolas públicas de tempo integral. São Paulo, Brasil. Archivos Latinoamericanos de Nutrición, 48:18-24.

SOUZA, F. J. P. S., 1992. Pobreza, Desnutrição e Mortalidade Infantil: Condicionantes Socioeconômicos. Fortaleza: UNICEF.

STATA CORPORATION, 1997. Stata Statistical Software: Realease 5.0. College Station: Stata Corporation.

STATISTICS AND EPIDEMIOLOGY RESEARCH CORPORATION, 1988. Egret. Epidemiology Graphics Estimation and Testing Package. Washington, D.C.: Statistics and Epidemiology Research Corporation.

TSUYUOKA, R.; BAILEY, J. W.; GUIMARÃES, A. M. D. N.; GURGEL, R. Q. \& QUEVAS, L. E., 1999. Anemia and intestinal parasitic infections in primary school students in Aracaju, Sergipe, Brazil. Cadernos de Saúde pública, 15:413-421.

VALENTE, F. L., 1986. Fome e Desnutrição: Determinantes Sociais. São Paulo: Cortez.

VÁZQUEZ, M. L.; MOSQUERA, M.; CUEVAS, L. E.; GONZALES, E. S.; VERAS, I. C. L.; LUZ, E. O.; BATISTA-FILHO, M. \& GURGEL, R. O., 1999. Incidência e fatores de risco de diarréia e infecções respiratórias agudas em comunidades urbanas de Pernambuco, Brasil. Caderno de Saúde Pública, 15:163-171.

VICTORA, C. G.; HUTLY, S. R.; FUCHS, S. C. \& OLINTO, M. T. A., 1997. The role of conceptual frameworks in epidemiological analysis: A hierarchical approach. International Journal of Epidemiology, 26:224-227.

VICTORA, C. G.; SMITH, P. G. \& VAUGHAN, J. P., 1989. Epidemiologia da Desigualdade. 2a Ed. São Paulo: Editora Hucitec/Edusp.

WHO (World Health Organization), 1980. The incidence of low birthweight: A critical review of available information. World Health Statistics Quarterly, 33:197-244.

WHO (World Health Organization), 1995. World Expert Committee on Physical Status: The Use and Interpretation of Anthropometry, 1993. WHO Technical Report Series 854. Geneva: WHO. 